\title{
The Static Stress Intensity Factor around the Antiplane Crack in an Infinite FGM Strip
}

\author{
Wen-bin Zhao, Zhi-xin Hu, Xue-xia Zhang, and Hai-ling Xie \\ School of Applied Science, Taiyuan University of Science and Technology, Taiyuan 030024, China \\ Correspondence should be addressed to Xue-xia Zhang; xuexiaz@126.com
}

Received 16 April 2013; Accepted 28 May 2013

Academic Editor: Marcelo Moreira Cavalcanti

Copyright (C) 2013 Wen-bin Zhao et al. This is an open access article distributed under the Creative Commons Attribution License, which permits unrestricted use, distribution, and reproduction in any medium, provided the original work is properly cited.

\begin{abstract}
The problem of the static stress intensity factor around antiplane crack in an infinite strip functionally graded material was studied by using the method of integral transform-dual integral equations in this paper. The shear modulus in the two principal directions of the functionally graded material was assumed to vary proportionately as gradient model of double parameters index function. The partial differential equation was first reduced to Euler equation with Fourier cosine transform. By solving dual integral equations that were derived by applying the solution of Euler equation with the method of Copson, stress intensity factor around the crack tip was derived. And the variation curves of the dimensionless stress intensity factor with the strip height, crack length, gradient parameter, and inhomogeneous coefficient are obtained by using the numerical calculation.
\end{abstract}

\section{Introduction}

Functionally gradient material is a new type of materials. The advent of functionally gradient material is in order to meet performance requirements of parts in all kinds of special environment. The sudden changes of the material composition and properties in a member often lead to obvious local stress concentration. If a material transition to another kind of material is the step by step, the stress concentration will greatly reduce. A new type of functional gradient materials (FGM) was developed in order to reduce the stress concentration of the material and improve the performance requirements of the material as discussed elsewhere [1-5]. Most previous studies about FGM in the past were focused on isotropic material as discussed elsewhere [6, 7]. However, a few FGMs were isotropic material. At present, the application of FGM has been expanded to space, energy, traffic, optics, chemistry, biomedical engineering, and so forth. However, due to the influence of the process conditions, FGM will produce some defects in manufacturing process. These defects will inevitably form crack due to stress action and crack expansion will affect the whole member. So, the research about crack and the crack expansion of FGM are very important as discussed elsewhere [8-11].

In the fracture mechanics study of FGM, most scholars use the power functions model as a material property parameter model. The model of $\mu(z)=\mu_{0}(1+c|z|)$ as discussed by Smith [1], the model of $\mu(z)=\mu_{0}(1+\alpha|z|)^{2}$ as discussed by Smith [12], and the model of $\mu(z)=\mu_{0}(1+\alpha|z|)^{k}$ as discussed by Smith [13]. However, the models of double parameters index function were adopted in this paper.

Usually the method of studies about stress intensity factor of antiplane crack in FGM is integral transforms-dualintegral equations. At present, most scholars put the partial differential equation into the Bessel equation with Fourier cosine transform in a designed material property parameter model. Then dual integral equations were obtained by using the solution of Bessel equation. But the difference between this paper and above references is that the partial differential equation is first reduced to Euler equation with Fourier cosine transform, and then dual integral equations were obtained by using the solution of Euler equation. Finally the local stress field and stress intensity factor around the crack tip were derived. This method is very few in the study of FGM. 


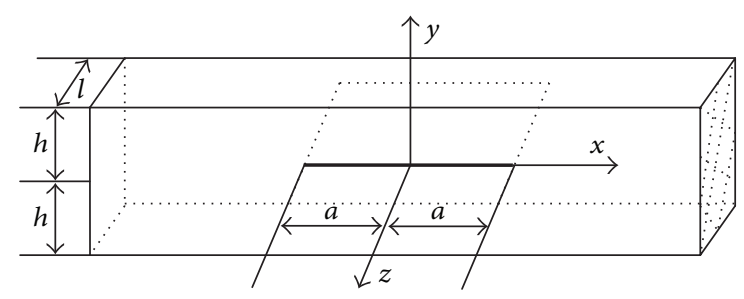

Figure 1: The model of crack in infinite strip functionally graded material.

\section{Formulation of the Problem}

Consider the antiplane problem of an infinite strip functionally graded material containing a crack of length $2 a$, and the thickness is $2 h$, as shown in Figure 1 . The $x$-axis and $y$-axis are the elastic principal axis.

Shear moduli $\mu_{x}, \mu_{y}$ are the functions of $y$, and $\mu_{x}, \mu_{y}$ vary according to the following functions:

$$
\begin{aligned}
& \mu_{x}(y)=\left(\mu_{x}\right)_{0} \exp (2 k \alpha y), \\
& \mu_{y}(y)=\left(\mu_{y}\right)_{0} \exp (2 k \alpha y),
\end{aligned}
$$

where parameter $\alpha>0, k>0$, the shear modulus $\left(\mu_{x}\right)_{0}$ and $\left(\mu_{y}\right)_{0}$, can be determined as $y=0$.

Suppose that $u_{x}, u_{y}$, and $u_{z}$ are displacement components of the direction $x, y$ and $z$. For the antiplane shear deformation, $u_{x}, u_{y}$ are 0 everywhere.

Assume that $u_{z}=\omega(x, y)$ and $\omega(x, y)$ is the displacement function. The relationship between the displacement function $\omega(x, y)$ and stress components $\tau_{x z}$ and $\tau_{y z}$ is written as

$$
\tau_{x z}=\mu_{x} \frac{\partial \omega}{\partial x}, \quad \tau_{y z}=\mu_{y} \frac{\partial \omega}{\partial y} .
$$

The stress equilibrium equation can be expressed as

$$
\frac{\partial \tau_{x z}}{\partial x}+\frac{\partial \tau_{y z}}{\partial y}=0
$$

We can obtain the governing equation [14-16] of antiplane problem in infinite strip functionally graded materials by substituting stress components (2) into (3):

$$
\frac{\partial^{2} \omega}{\partial x^{2}}+\frac{\mu_{y}}{\mu_{x}} \frac{\partial^{2} \omega}{\partial y^{2}}+\frac{\mu_{y}^{\prime}}{\mu_{x}} \frac{\partial \omega}{\partial y}=0
$$

The corresponding boundary conditions of the crack surface under antiplane shear loading $\tau_{0}$ are as follows:

$$
\begin{array}{cc}
\omega(x, 0)=0, & |x|>a, \\
\tau_{y z}(x, 0)=-\tau_{0}, & |x| \leq a, \\
\tau_{x z}(x, \pm h)=0, & |x|<\infty .
\end{array}
$$

Therefore, an analysis of antiplane problem in infinite strip functionally graded materials is to solve the boundary value problem of partial differential equation (4)-(7).

\section{Dual Integral Equation}

Due to the symmetry, we only need to study the part of $x>0$, $y>0$. Introducing the following Fourier cosine transform:

$$
\begin{gathered}
\tilde{f}(s)=\int_{0}^{\infty} f(x) \cos (s x) d x, \\
f(x)=\frac{2}{\pi} \int_{0}^{\infty} \tilde{f}(s) \cos (s x) d s,
\end{gathered}
$$

where $\tilde{f}(s)$ is called Fourier cosine transform of $f(x)$, and $f(x)$ is called Fourier cosine inverse transform of $\widetilde{f}(s)$.

Define

$$
\omega(x, y)=\frac{2}{\pi} \int_{0}^{\infty} U(s, y) \cos (s x) d s .
$$

Among them, $U(s, y)$ is the Fourier integral cosine transformation function of displacement function $\omega(x, y)$.

By using the Fourier integral transformation, (4) can be converted into as follows:

$$
\frac{\partial^{2} U(s, y)}{\partial y^{2}}+2 k \alpha \frac{\partial U(s, y)}{\partial y}-S^{2} U(s, y)=0,
$$

where $S=s r$, and inhomogenous coefficient $r=$ $\sqrt{\left(\mu_{x}\right)_{0} /\left(\mu_{y}\right)_{0}}$.

Choosing proper variable substitutions as follows:

$$
Y=(\exp (\alpha y))^{(k+1) / 2} U, \quad X=S \exp (\alpha y) .
$$

So

$$
\begin{aligned}
\frac{\partial U}{\partial y}= & S^{(k+1) / 2} X^{-(k+1) / 2} \frac{d Y}{d y} \\
& -\frac{k+1}{2} \alpha S^{(k+3) / 2} X^{-(k+3) / 2} \exp (\alpha y) Y,
\end{aligned}
$$

$$
\begin{aligned}
\frac{\partial^{2} U}{\partial y^{2}}= & S^{(k+1) / 2} X^{-(k+1) / 2} \frac{d^{2} Y}{d y^{2}} \\
- & (k+1) \alpha S^{(k+3) / 2} X^{-(k+3) / 2} \exp (\alpha y) \frac{d Y}{d y} \\
+ & \frac{k+1}{2}\left(\frac{k+3}{2} S^{(k+5) / 2} X^{-(k+5) / 2} \alpha^{2} \exp (2 \alpha y)\right. \\
- & \left.S^{(k+3) / 2} X^{-(k+3) / 2} \alpha^{2} \exp (\alpha y)\right) Y \\
\frac{d Y}{d y} & =S \alpha \exp (\alpha y) \frac{d Y}{d X}
\end{aligned}
$$

$$
\frac{d^{2} Y}{d y^{2}}=S \alpha^{2} \exp (\alpha y) \frac{d Y}{d X}+S^{2} \alpha^{2} \exp (2 \alpha y) \frac{d^{2} Y}{d X^{2}}
$$

By substituting (12) into (10), partial differential equation (10) can be translated into Euler equation as follows:

$$
X^{2} \frac{d^{2} Y}{d X^{2}}+k X \frac{d Y}{d X}+\beta^{2} Y=0
$$


where

$$
\beta=\sqrt{\frac{(1+k)(1-3 k)}{4}-\frac{S^{2}}{\alpha^{2}}} .
$$

Introducing transformations of the independent variable

$$
X=\exp (t), \quad t=\ln (X) .
$$

So, (13) can be transformed into the following formula:

$$
\frac{d^{2} Y}{d t^{2}}+(k-1) \frac{d Y}{d t}+\beta^{2} Y=0 .
$$

The general solution of Euler equation (16) can be written as

$$
\begin{aligned}
Y= & C_{1} \exp \left(\frac{(1-k)+\sqrt{(k-1)^{2}-4 \beta^{2}}}{2} \cdot t\right) \\
& +C_{2} \exp \left(\frac{(1-k)-\sqrt{(k-1)^{2}-4 \beta^{2}}}{2} \cdot t\right) \\
= & C_{1} X^{\left((1-k)+\sqrt{\left.(k-1)^{2}-4 \beta^{2}\right)} / 2\right.}+C_{2} X^{\left((1-k)-\sqrt{\left.(k-1)^{2}-4 \beta^{2}\right)} / 2\right.},
\end{aligned}
$$

where $C_{1}$ and $C_{2}$ are the coefficients of solution.

Substituting (17) into (11), we can obtain the formula of solution as follows:

$$
\begin{aligned}
U(s, y)= & A_{1}(s) \exp \left[\alpha\left(k_{1}-\frac{k+1}{2}\right) y\right] \\
& +A_{2}(s) \exp \left[\alpha\left(k_{2}-\frac{k+1}{2}\right) y\right],
\end{aligned}
$$

where

$$
\begin{aligned}
& A_{1}(s)=C_{1} S^{\left((1-k)+\sqrt{(k-1)^{2}-4 \beta^{2}}\right) / 2}, \\
& A_{2}(s)=C_{2} S^{\left((1-k)-\sqrt{(k-1)^{2}-4 \beta^{2}}\right) / 2}, \\
& k_{1}=\frac{(1-k)+\sqrt{(k-1)^{2}-4 \beta^{2}}}{2}, \\
& k_{2}=\frac{(1-k)-\sqrt{(k-1)^{2}-4 \beta^{2}}}{2} .
\end{aligned}
$$

From (9) and (18), the integral expression of displacement function was obtained as follow:

$$
\begin{aligned}
& \omega(x, y) \\
& \begin{aligned}
=\frac{2}{\pi} \int_{0}^{\infty} & \left\{A_{1}(s) \exp \left[\alpha\left(k_{1}-\frac{k+1}{2}\right) y\right]\right. \\
& \left.+A_{2}(s) \exp \left[\alpha\left(k_{2}-\frac{k+1}{2}\right) y\right]\right\} \cos (s x) d s .
\end{aligned}
\end{aligned}
$$

The shear stresses $\tau_{x z}$ and $\tau_{y z}$ can be obtained from (20) and the relationship of stress-displacement (2). The stress formulas are listed as follows:

$$
\begin{gathered}
\tau_{y z}(x, y) \\
=\mu_{y} \frac{\partial \omega}{\partial y}=\frac{2}{\pi} \mu_{y} \\
\cdot \int_{0}^{\infty}\left\{\alpha\left(k_{1}-\frac{k+1}{2}\right) A_{1}(s)\right. \\
\cdot \exp \left[\alpha\left(k_{1}-\frac{k+1}{2}\right) y\right] \\
+\alpha\left(k_{2}-\frac{k+1}{2}\right) \cdot A_{2}(s) \\
\left.\cdot \exp \left[\alpha\left(k_{2}-\frac{k+1}{2}\right) y\right]\right\} \cos (s x) d s, \\
\tau_{x z}(x, y) \quad \mu_{x} \frac{\partial \omega}{\partial x}=-\frac{2}{\pi} \mu_{x} \\
\cdot \int_{0}^{\infty} s\left\{A_{1}(s) \exp \left[\alpha\left(k_{1}-\frac{k+1}{2}\right) y\right]\right. \\
\left.\quad+A_{2}(s) \exp \left[\alpha\left(k_{2}-\frac{k+1}{2}\right) y\right]\right\}
\end{gathered}
$$

$$
\times \sin (s x) d s
$$

We can obtain the relation between $A_{1}(s)$ and $A_{2}(s)$ by substituting (22) into the boundary conditions (7) as follows:

$$
A_{1}(s)=-A_{2}(s) \exp \left[-2 \alpha h \sqrt{k^{2}+\frac{s^{2} r^{2}}{\alpha^{2}}}\right] \text {. }
$$

Let

$$
\begin{aligned}
& A_{1}(s)=-\frac{\exp \left[-2 \alpha h \sqrt{k^{2}+s^{2} r^{2} / \alpha^{2}}\right]}{1-\exp \left[-2 \alpha h \sqrt{k^{2}+s^{2} r^{2} / \alpha^{2}}\right]} A(s), \\
& A_{2}(s)=\frac{1}{1-\exp \left[-2 \alpha h \sqrt{k^{2}+s^{2} r^{2} / \alpha^{2}}\right]} A(s) .
\end{aligned}
$$

From (20), (21), (24), and boundary conditions (5) and (6), we can obtain the dual integral equations:

$$
\begin{gathered}
\int_{0}^{\infty} A(s) \cos (s x) d s=0, \quad x>a, \\
\int_{0}^{\infty} s A(s) G(s) \cos (s x) d s=\frac{\pi \tau_{0}}{2 r\left(\mu_{y}\right)_{0}}, \quad 0<x \leq a,
\end{gathered}
$$


in which,

$$
\begin{aligned}
G(s)= & -\alpha\left(k_{2}-\frac{k+1}{2}\right)+\alpha\left(k_{1}-\frac{k+1}{2}\right) \\
& \left.\times \exp \left[-2 \alpha h \sqrt{k^{2}+\frac{s^{2} r^{2}}{\alpha^{2}}}\right]\right) \\
& \times\left(\operatorname{sr}\left(1-\exp \left[-2 \alpha h \sqrt{k^{2}+\frac{s^{2} r^{2}}{\alpha^{2}}}\right]\right)\right)^{-1} .
\end{aligned}
$$

Solving the dual integral equations (25a) and (25b) by using Copson-Sih method [17], the solution can be expressed as

$$
A(s)=\frac{\pi \tau_{0} a^{2}}{2 r\left(\mu_{y}\right)_{0}} \int_{0}^{1} \sqrt{\xi} \Phi(\xi) J_{0}(s a \xi) d \xi,
$$

where $J_{0}(\cdot)$ is the so-called zero Bessel function of the first kind. The function $\psi(\xi)$ can be governed by the Fredholm integral equation of the second kind:

$$
\Phi(\xi)+\int_{0}^{1} \Phi(\eta) M(\xi, \eta) d \eta=\sqrt{\xi}
$$

where kernel function $M(\xi, \eta)$ is

$$
M(\xi, \eta)=\sqrt{\xi \eta} \int_{0}^{\infty} s\left[G\left(\frac{s}{a}\right)-1\right] J_{0}(s \xi) J_{0}(s \eta) d s
$$

By using numerical integral method, the numerical solution of $\Phi(1)$ can be given from (28) and (29).

\section{The Stress Intensity Factor}

By using of the formula of integration by parts, formula (27) can be written as

$$
\begin{aligned}
A(s)= & \frac{\pi \tau_{0}}{2 r\left(\mu_{y}\right)_{0}} \frac{a}{s} \\
& \times\left[\Phi(1) J_{1}(s a)-\int_{0}^{1} \xi J_{1}(s a \xi) \frac{d\left(\xi^{-1 / 2} \Phi(\xi)\right)}{d \xi} d \xi\right]
\end{aligned}
$$

We can obtain the stress principle by substituting (30) into (21)-(24) as follows:

$$
\begin{gathered}
\tau_{y z}(x, 0)=\frac{\tau_{0} a \Phi(1)}{r} \int_{0}^{\infty} H(s) J_{1}(s a) \cos (s x) d s \\
\tau_{x z}(x, 0)=-r \tau_{0} a \Phi(1) \int_{0}^{\infty} J_{1}(s a) \sin (s x) d s
\end{gathered}
$$

In formula (31),

$$
\begin{aligned}
H(s)= & \frac{-\alpha\left(k_{1}-(k+1) / 2\right) \exp \left(-2 \alpha h \sqrt{k^{2}+s^{2} r^{2} / \alpha^{2}}\right)}{s\left[1-\exp \left(-2 \alpha h \sqrt{k^{2}+s^{2} r^{2} / \alpha^{2}}\right)\right]} \\
& +\frac{\alpha\left(k_{2}-(k+1) / 2\right)}{s\left[1-\exp \left(-2 \alpha h \sqrt{k^{2}+s^{2} r^{2} / \alpha^{2}}\right)\right]} .
\end{aligned}
$$

It is noticed that

$$
H(s)=-r, \quad \text { as } s \longrightarrow \infty .
$$

Considering the stress singularity around crack tip as $s \rightarrow$ $\infty$, the stress principle can be written as

$$
\begin{gathered}
\tau_{y z}(x, 0)=-\tau_{0} a \Phi(1) \int_{0}^{\infty} J_{1}(s a) \cos (s x) d s, \\
\tau_{x z}(x, 0)=-r \tau_{0} a \Phi(1) \int_{0}^{\infty} J_{1}(s a) \sin (s x) d s .
\end{gathered}
$$

Introducing the complex transformations as follows:

$$
z=x+i y=r e^{i \theta}, \quad z-a=r_{1} e^{i \theta_{1}}, \quad z+a=r_{2} e^{i \theta_{2}} .
$$

For

$$
\int_{0}^{\infty} J_{1}(s a) \cos (s x) d s=-\frac{x}{a \sqrt{(x-a)(x+a)}} .
$$

The formula (35) can be simplified to

$$
\tau_{y z}(x, 0)=\frac{\tau_{0} \Phi(1) x}{\sqrt{(x-a)(x+a)}} .
$$

Defining the stress intensity factor as follows:

$$
K_{\mathrm{III}}=\lim _{x \rightarrow a^{+}} \sqrt{2 \pi(x-a)} \tau_{y z}(x, 0) .
$$

So, we can obtain

$$
K_{\mathrm{III}}=\tau_{0} \sqrt{\pi a} \Phi(1)
$$

Formula (41) can be written as

$$
\Phi(1)=\frac{K_{\mathrm{III}}}{\tau_{0} \sqrt{\pi a}},
$$

where $\Phi(1)$ is called dimensionless stress intensity factor.

The variation curves of the dimensionless stress intensity factor $\Phi(1)$ with the strip height $h$, crack length $a$, gradient parameter are $k$ and $\alpha$, and inhomogenous coefficient $r$ are obtained by using the numerical calculation as shown in Figures 2-4.

Figure 2 shows the variation curves of the dimensionless stress intensity factor with strip height and crack length under the same condition of inhomogeneous coefficient and gradient parameter. It is noticed that the longer crack and the thinner strip lead to the greater the stress intensity factor. 


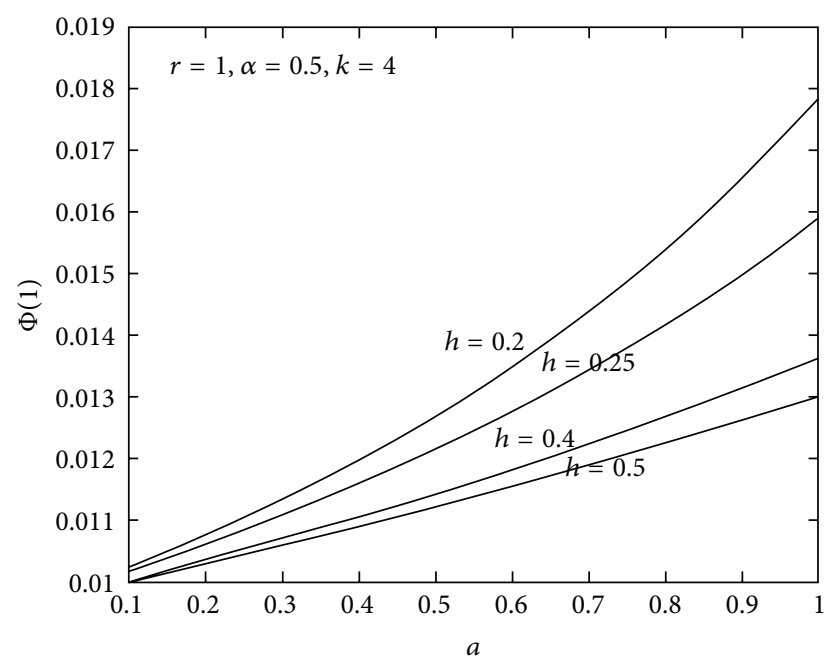

FIGURE 2: The effect of strip height $h$ and crack length $a$ on the stress intensity factor $\Phi(1)$.

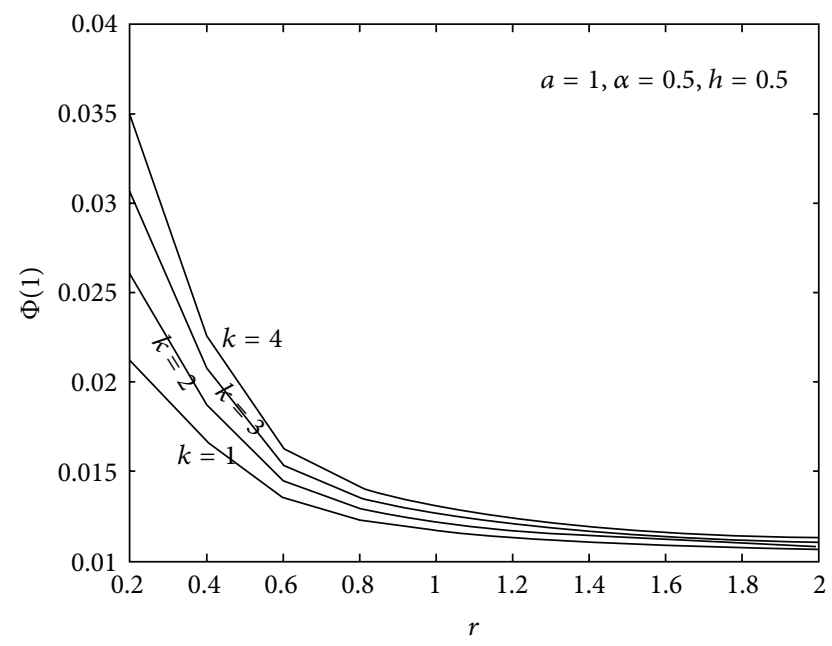

FIGURE 3: The effect of gradient parameter $k$ and inhomogenous coefficient $r$ on the stress intensity factor $\Phi(1)$.

Figure 3 shows the variation curves of the dimensionless stress intensity factor with inhomogeneous coefficient and gradient parameter $k$ under the same condition of strip height, crack length, and gradient parameter $\alpha$. It is noticed that the greater gradient parameter $k$ and the smaller inhomogenous coefficient lead to the greater stress intensity factor.

Figure 4 shows the variation curves of the dimensionless stress intensity factor with crack length and gradient parameter $\alpha$ under the same condition of strip height, inhomogenous coefficient, and gradient parameter $k$. It is noticed that the greater gradient parameter $\alpha$ and the longer crack lead to the greater of the stress intensity factor.

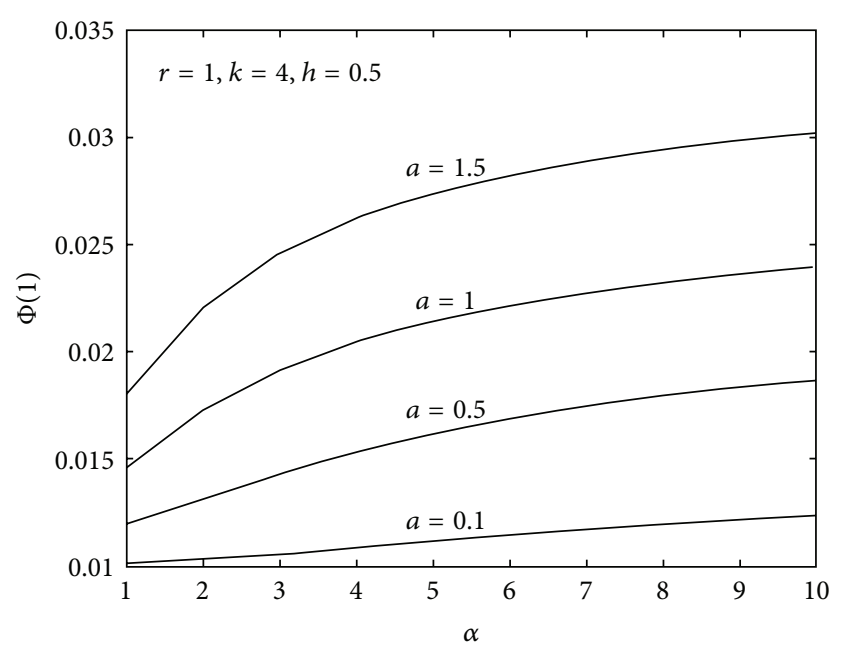

FIgURE 4: The effect of gradient parameter $\alpha$ and crack length $a$ on the stress intensity factor $\Phi(1)$.

\section{Conclusions}

(1) There is $r^{-1 / 2}$ singularity in crack tip of the orthotropic functionally graded material. It is the same as the orthotropic homogeneous material. Thus, we can study the complex fracture problem of the orthotropic functionally graded material in using the conventional method fracture mechanics.

(2) Figure 2 shows the stress intensity factor $\Phi(1)$ increase with the increasing of crack length $a$ in the condition of fixed strip height $h$. The stress intensity factor $\Phi(1)$ decreases with the increasing of strip height $h$ in the condition of fixed crack length $a$.

(3) Figure 3 shows the stress intensity factor $\Phi(1)$ decrease with the increasing of inhomogenous coefficient $r$ in the condition of fixed gradient parameter $k$, The stress intensity factor $\Phi(1)$ increases with the increasing of gradient parameter $k$ in the condition of fixed inhomogenous coefficient $r$.

(4) Figure 4 shows the stress intensity factor $\Phi(1)$ increase with the increasing of gradient parameter $\alpha$ in the condition of fixed crack length $a$. The stress intensity factor $\Phi(1)$ increases with the increasing of crack length $a$ in the condition of fixed gradient parameter $\alpha$.

\section{Acknowledgments}

Project supported by the Science and Technology Major Project of the Ministry of Education of China (208022) and the Graduate Scientific and Technological Innovation Project of the Taiyuan University of Science and Technology (20122005 and 20125027). 


\section{References}

[1] F. Erdogan, "The crack problem for bonded nonhomogeneous materials under antiplane shear loading," Journal of Applied Mechanics, vol. 52, no. 4, pp. 823-828, 1985.

[2] N. Konda and F. Erdogan, "The mixed mode crack problem in a nonhomogeneous elastic medium," Engineering Fracture Mechanics, vol. 47, no. 4, pp. 533-545, 1994.

[3] F. Erdogan, "Fracture mechanics of functionally graded materials," Composites Engineering, vol. 5, no. 7, pp. 753-770, 1995.

[4] R. Mahajan, F. Erdogan, B. Kilic, and E. Madenci, "Cracking of an orthotropic substrate reinforced by an orthotropic plate," International Journal of Solids and Structures, vol. 40, no. 23, pp. 6389-6415, 2003.

[5] M. A. Guler and F. Erdogan, "The frictional sliding contact problems of rigid parabolic and cylindrical stamps on graded coatings," International Journal of Mechanical Sciences, vol. 49, no. 2, pp. 161-182, 2007.

[6] G. C. Sih, "Crack tip mechanics based on progressive damage of arrow: hierarchy of singularities and multiscale segments," Theoretical and Applied Fracture Mechanics, vol. 51, no. 1, pp. 1132, 2009.

[7] G. C. Sih and K. K. Tang, "Assurance of reliable time limits in fatigue depending on choice of failure simulation: energy density versus stress intensity," Theoretical and Applied Fracture Mechanics, vol. 55, no. 1, pp. 39-51, 2011.

[8] A. M. Afsar, N. M. L. Huq, and J. I. Song, "Analytical solution to a mixed boundary value elastic problem of a roller-guided panel of laminated composite," Archive of Applied Mechanics, vol. 80, no. 4, pp. 401-412, 2010.

[9] E. Asadi and S. J. Fariborz, "Free vibration of composite plates with mixed boundary conditions based on higher-order shear deformation theory," Archive of Applied Mechanics, vol. 82, no. 6, pp. 755-766, 2012.

[10] V. Parameswaran and A. Shukla, "Crack-tip stress fields for dynamic fracture in functionally gradient materials," Mechanics of Materials, vol. 31, no. 9, pp. 579-596, 1999.

[11] A. Gerasoulis and R. P. Srivastav, "A Griffith crack problem for a nonhomogeneous medium," International Journal of Engineering Science, vol. 18, no. 1, pp. 239-247, 1980.

[12] C. Y. Li, Z. Zhenzhu, and D. Zhuping, "Dynamic stress field around the mode III crack tip in an orthotropic functionally graded material," Applied Mathematics and Mechanics, vol. 21, no. 6, pp. 651-658, 2000.

[13] C. Y. Li, Z. Z. Zou, and Z. P. Duan, "Stress filed around the antiplane crack tip in an orthotropic functionally graded material," Mechanics of Solids, vol. 22, no. 1, pp. 81-84, 2001 (Chinese).

[14] X. S. Bi, J. Cheng, and X. L. Chen, "The problem of anti-plane crack in infinite strip functionally gradient material," Journal of Harbin Industrial University, vol. 34, no. 3, pp. 363-366, 2002.

[15] X. G. Li, X. S. Bi, and D. Y. Chu, "The problem of movement crack in infinite strip anisotropic functionally gradient material," Journal of Liaoning Engineering Technology University, vol. 26, no. 6, pp. 847-849, 2007.

[16] Z. Q. Cheng and Z. Zhong, "The problem of type III movement crack in functionally gradient material," Journal of Applied Mechanics, vol. 25, no. 1, pp. 62-65, 2008.

[17] E. T. Copson, "On certain dual integral equations," Proceedings of the Glasgow Mathematical Association, vol. 5, no. 1, pp. 21-24, 1961. 


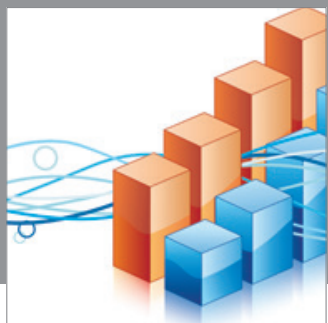

Advances in

Operations Research

mansans

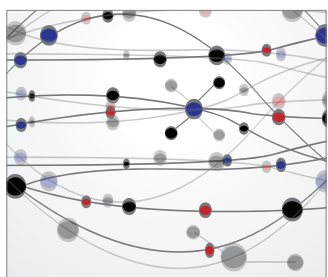

The Scientific World Journal
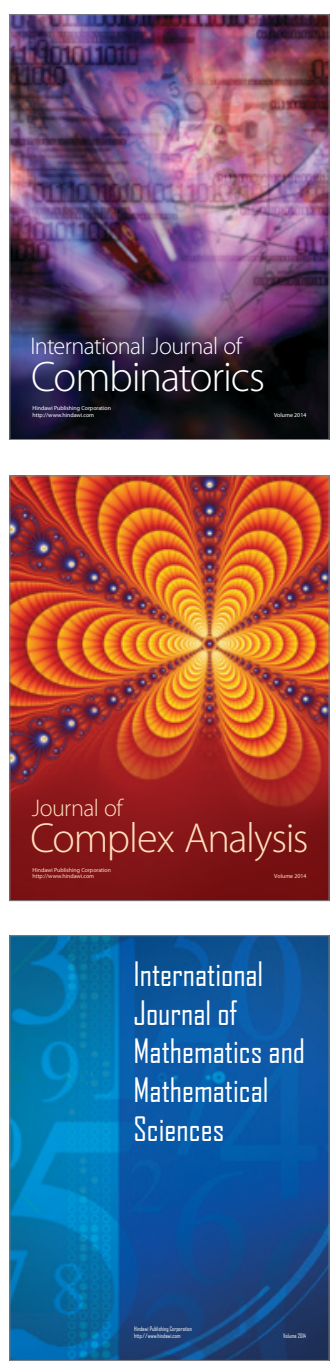
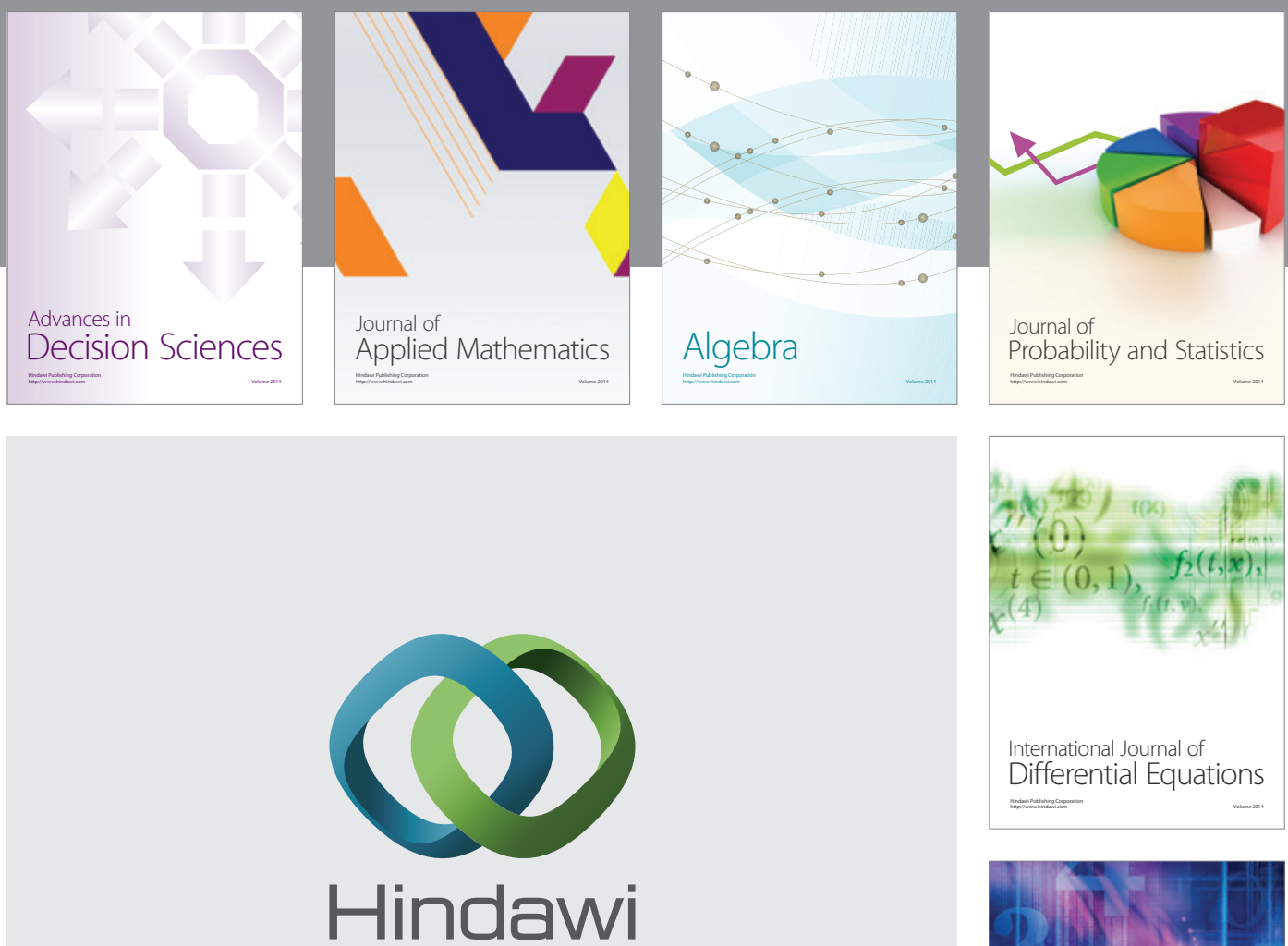

Submit your manuscripts at http://www.hindawi.com
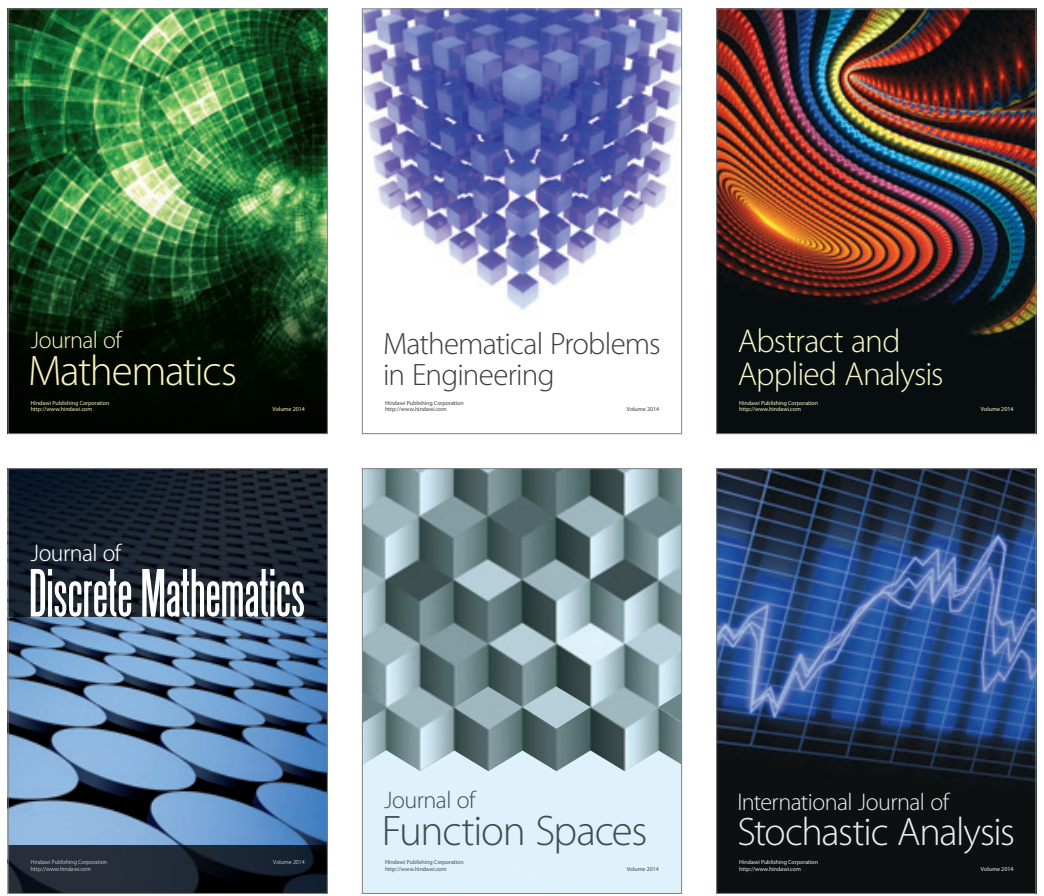

Journal of

Function Spaces

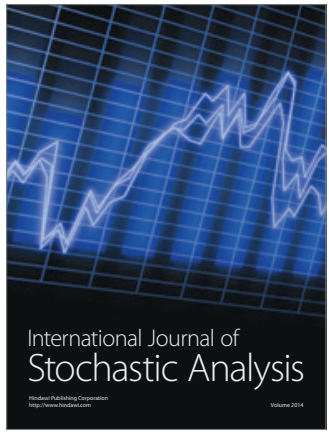

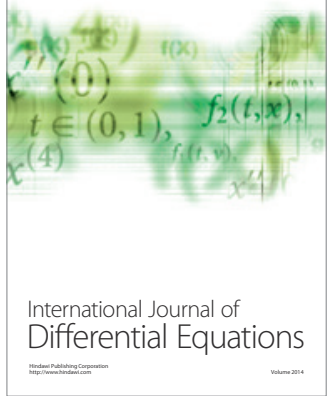
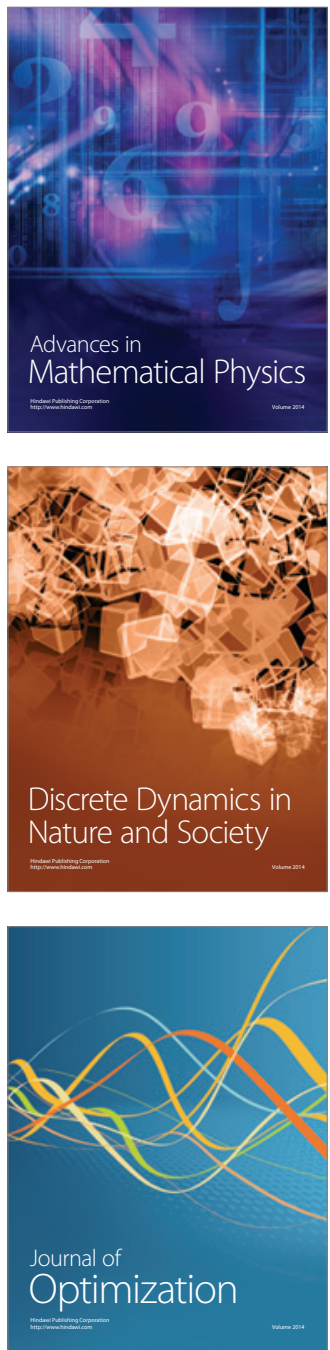